\title{
MORFOLOGIA DAS CRÓNICAS PORTUGUESAS DOS P.M.H. (artigos, numerais e pronomes)
}

1. Dos cronicões dos Portugaliae Monumenta Historica (vol. Scriptores), Lisboa, 1856, publicados sob a direç̧ão do insigne historiador Alexandre Herculano, vamos estudar agora a parte da morfologia que abrange os artigos, os numerais e os pronomes.

Muitos outros aspectos já foram por nós tratados quer em artigos, quer em comunicações, quer em livros, o último dos quais intitulado Crónica da Tomada de Lisboa (Lisboa, 1995).

A grafia empregada é a dos originais, quando existem, ou a dos mais antigos apógrafos; no entanto, para facilitar a localização dos vocábulos, apresentamos aquela em que se acham nos Scriptores (página, coluna e linha), excepto nas ocorrências repetidas. Sempre que os termos estudados não se usam na actualidade, levam a indicação de antigos (ant.) ou arcai$\cos$ (arc., estes não posteriores a 1500).

2. Como é normal, começamos pelos artigos definidos e suas contraç̧ões ou combinações com diversas preposições. Masculino do singular: o, "des Ocomeço" (22, A, 8); el- /عl/, "ElRey noso señor" (22, A, 22); "Estando assi elRey" (408, A, 62); feminino do singular: a, "he arrenenbrança" (22, A, 17); la, arc., ainda popular, "por lla emveja que aujam" (76, B, 25); plural masc.: os, "os seus Juizes" (22, A, 23); fem.: as, "as maiores casas" $(25, \mathrm{~B}, 11)$; variantes arcaicas do artigo lo, la, los, las (formas primitivas): masc. sing.: no, "em no corpo" (75, B, 45); fem. sing.: na, "ẽ na era" (29, A, 47); masc. plural: nos, "em nos seus feitos" (75, B, 38); fem. plur.: nas "em nas maaos" (78, A, 13). Contracções/combinações:

a) com a preposição $\boldsymbol{a}$ : fem. sing.: à, "à ujda" (75, B, 30); aa, arc., "aasua custa" (22, A, 54); masc. sing.: ao, "ao papa" (22, B, 49); masc. plur.: aos, "aos mouros" (24, A, 36); òs, arc. e pop., "preguntou affomso Enrriquez os (=òs) uassalos" (29, B, 41); fem. plur.: às, "as (=às) horas canonicas" (409, B, 18);

b) com a prep. com: masc. sing.: coo, "coo conde" (29, B, 51); fem. sing.: coa, "coa Rainha" (31, B, 24);

c) com a prep. de: masc. sing.: do, "do algarue" (22, A, 18); fem. sing.: da, "da Coroa" (22, A, 25); masc. plur.: dos, "dos Reyes" (22, A, 17); fem. plur.: das, "das bestas" (27, B, 7);

d) com a prep. dês: masc. plur., ant. e pop.: delo, "delo começo" (414, A, 19);

e) com a prep. em: masc. sing.: no, "no dĩcto logar" (22, A, 40); fem. sing.: na, "na maneira" (22, A, 33); masc. plur.: nos, "nos liuros" (407, A, 39); fem. plur.: nas, "na[s] azaas" (29, B, 67); 
f) com a prep. per (arcaizante): masc. sing.: pelo, "pello bispo" (27, B, 70); fem. sing.: pela, "pella porta" (27, A, 16); masc. plur.: pelos, "pelos merecimẽtos"(410, A, 25); fem. plur.: pelas, "pellas mããos" (25, A, 55);

g) com a prep. por: masc. sing.: polo, ant., "polo seu sancto acordo" (407, B, 32); fem. sing.: pola, ant., "pola uida" (409, A, 2); masc. plur.: polos, ant., "pollos peccadores" (24, A, 17); fem. plur.: polas, ant., "polas almas" (409, B, 11).

3. Artigos indefinidos: masc. sing.: um, "em hum acordo" (416, A, 42) [hum= $\tilde{u}$ (hoje um) não é anterior ao séc. XV, segundo José Leite de Vasconcelos]; ũu, arc., "hũu homẽ" $(407, \mathrm{~B}, 22)$ [até pelo menos ao séc. XV, de acordo com José Joaquim Nunes]; fem. sing.: ũa, ant. e pop., "hũa dona" $(22, \mathrm{~B}, 27)$ [até tarde na língua literária, na pop. ainda hoje se usa, por ex. na Madeira; uma só depois do séc. XVI (J. J. Nunes)]. Contraç̧ão ant. e pop. com a prep. de: dũa, fem. sing., ant., "natural dhũa uila a que dizem bõna" $(410, \mathrm{~A}, 3)$ [hoje é só duma, pl. dumas (ant. dũas) e masc. sing.: dum, pl. duns, e é sempre lícito não usar as formas contractas, mas de um, de uma, de uns, de umas]. Artigo partitivo: masc. plur.: dos, arc., "dos Mourros que ficarom se acolherom ao alcaçar".

\section{Numerais:}

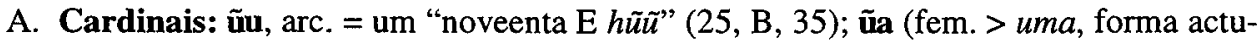
al), "hũa cruz de prata" (24, B, 60); dous (forma desusada, por dois), "dous años" (22, A, 50); duas, fem., "duas vezes" (24, B, 18); três, "tres años" (23, B, 6); quatro, "quatro años" (22, B, 41); cinco, "as cinquo qujnas" (30, A, 39); cínqui, arc., "çinqui años" (22, B, 56); seis, "seis dias" (22, A, 51); sete, "sete años" (22, B, 43); oito, "oito años" (22, B, 32); nove, "nove años" (22, A, 21); dez, "pera dez años" (27, A, 19); onze, "onze dias" (22, B, 30); doze, "os doze pares" (24, A, 34); treze, "treze Reys mourros" (25, B, 24); 14 (falta); quinze, "qujnze dias" (78, B, 27); 16 (falta); dezessete (forma ainda usual no Brasil, por dezassete); "dezesete años" (23, A, 7); dezoito, "dez oito dias" (31, B, 69); 19 (falta); vinte, "vjnte E seis añños" (31, B, 57); viinte, arc., "vijnte e noue" (22, A, 21); trinta, "trinta e sete" (22, B, 43); triinta, arc., "trijnta e dous" (22, A, 50); quarenta, "quarenta e sete" (407, B, 44); quareenta, arc., "quareenta e noue" (22, B, 33); corenta, ant. e pop., "quorenta Ecĩquo" (32, B, 4); cinquenta, "Cinquoenta años" (22, A, 41); sesseenta, arc., "seseenta e tres años" (23, B, 6); sasseenta, arc., "saseenta e hũũ años" (22, B, 42); seteenta, arc., "seteenta annos" (24, A, 16); oiteenta, arc., "oiteenta e çinqui" (22, B, 56); noveenta, arc., "noueenta e hũũ" (22, A, 52); cento ..., "Çento e Çinquoenta" (22, A, 40); duzentos, "mjle dozentos" (24, B, 42); trezentos, "mjl e trezentos" (23, A, 8); quatrocentos, "mjlequatroçentos" (22, A, 20); quinhentos, "qujnhêtos maraujdijes" (77, A, 74); novecentos, "nouecentos annos" (24, A, 33); mil, "mjlequatroçentos" (22, A, 20).

B. Ordinais: primeiro, masc., "oprimeiro Rey" (24, A, 72); primeira, fem., "a prime $i$ ra pedra" (409, A, 17); segundo, "filho segundo" (24, B, 75); segunda, "a outra pedra segü$d a$ " (409, A, 39); terceira, fem., "na terceira uez". (410, B, 26); treceiro, masc., arc. e pop., 
"o treçeiro ffoy dom pero paaez" (28, B, 70); quarto, "quarto Jdus" (78, A, 5); sexto, no fem. sexta, "sesta feira" (24, A, 8).

C. I. Colectivos: milhares, "mjlhares delles" (25, B, 64). II. Partitivos: meo, arc. (<> actual meio), "acabado meo año" (76, A, 14); "Em meeo do coro" (78, A, 16); meatade, arc. ( $<>$ actual metade), "o coytelo entrou pela meatade do dito pam" (410, B, 60). III. Distributivos: senhos, ant. (existe a variante sendos) < singulos, "acadahũu senhos pããẽs" $(24, \mathrm{~B}, 44)$.

\section{Pronomes.}

A. Pessoais: (a) formas tónicas de sujeito: $1^{\mathrm{a}}$ p. sing.: eu, "que $e u$ leixo" $(26, \mathrm{~A}, 46) ; 2^{\mathrm{a}}$ p. sing: tu, "tu perderias" (29, B, 34); "tu senhor es hũũ deus soo" (411, A, 26); 3" p. masc. sing.: ele (não ocorre); el, arc. e pop. /el/, "que ell fez" (23, B, 8); "Eell veendo que nom queriam fazer" (28, A, 8); fem. sing.: ela, "ella sse affastara" (26, A, 56); "E stando ella em o hospital" (25, A, 50); $1^{\mathrm{a}}$ p. plur.: nós, "vos ou nos ssairemos" $(26, \mathrm{~B}, 18)$; $2^{\mathrm{a}}$ p. plur.: vós, "ssairemos de purtugall ou uos" (26, B, 18); "Amigos uos bem sabedes" $(408, \mathrm{~B}, 11)$; $3^{\mathrm{a}} \mathrm{p}$. plur. masc.: eles; "elles lhe disserom" (26, A, 70); elas, fem. (falta); ende, arc. (=dele, dela, deles, delas), "esta terra que teeu lheixo deestroga ataa alem decoJnbra nom percas ende hũũ palmo" (29, B, 22); (b) formas tónicas com preposição: $1^{\text {a }}$ p. sing.: mim, "amy”" (26, A, 50); mi, ant., "cõ todo seu dotamẽto fique a $m i$ " $(412, \mathrm{~B}, 21) ; 2^{\mathrm{a}}$ p. sing.: ti, "ella se affastara de ty" (26, A, 56); $3^{\underline{a}}$ p. sing. masc.: ele, "que fose com elle" (75, B, 51); reflexo: si, "perasy E pera todos" (23, B, 9); “sentia ẽ sy" (410, A, 75); nós, $1^{\underline{a}}$ p. plur., "por nos" (76, B, 50); $2^{\mathrm{a}}$ p. plur.: vós (falta); $3^{\mathbf{a}}$ p. plur. fem.: elas, "em ellas faz mençõ" (22, A, 29); (c) formas átonas: $1^{\mathrm{a}}$ p. sing. complemento: me, "mandame ssoterrar" (26, A, 65); "dahonrra que me meu padre leixou" $(26, \mathrm{~B}, 47)$; $2^{\mathrm{a}} \mathrm{p}$. sing. compl.: te, "logo te torna" $(26, \mathrm{~A}, 63)$; "eu te hordeno" (28, A, 21); $3^{\mathrm{a}}$ p. sing. compl. ind.: lhe, "quelhe deus mandou" $(24, \mathrm{~A}, 7)$; masc. compl. dir.: o, "assy opodiam fazer" (24, B, 12); "que o metam ẽ sua oraçom" (24, B, 45); fem.: a, "pera $a$ defensarem" (24, B, 1); masc. plur.: os, "que os venceo" $(24, \mathrm{~A}, 69)$; fem. plur.: as, "as no podesse fazer" ( $25, \mathrm{~B}, 42)$; no $[<>l o>o]$, "honrauãno muito" (28, A, 30); "fezerõno [neutro] asi" (30, A, 52); na [<> la>a], "cobrarãna os mouros" (24, A, 6); reflexo: se, "quando sse finou" (22, A, 30); $1^{\text {a }}$ p. plur. compl.: nos, "nõ nos faz mester" $(26, \mathrm{~B}, 23)$; "mudemonos Em abito" (76, B, 69); 2a p. plur. compl.: vos, "escumungou uos" (30, B, 21); "segundo uos aestoria deuj-sara $(26, \mathrm{~B}, 51)$; $3^{\text {a }}$ p. plur. compl. dir. fem.: las, "exllas aqui" (27, B, 23); 3"a p. plur. compl. ind.: Ihes, "e que lhes desse hũu logar" (408, A, 43); lhe (=lhes), ant. e pop., "aos conçelhes [sic] ffazelhe honrra" (26, A, 51); "lhe demos sepulturas" (408, B, 36); lhis, arc., "Ihis demos logar" (408, B, 41); "pera lhis averẽ de dar os sacramẽtos" (409, B, 38); (d) compl. ind.+ compl. dir.: mo, "nom mo outorgou" (76, A, 60); ma, "dom affom[so] ma leixou" (26, B, 22); lho, "nõ tho quis consintir" $(26, \mathrm{~B}, 3)$; lha, "despois lha tomou o conde dom ffernando" (26, B, 4); lho, arc. [=-s+lo], "majs em modo o glorioso padre santo escrepueo ao duque de purtugall tam gloriosamente podemolho veer" (77, A, 33); no-lo, "ssegundo nollo Ja aestoria ha Ja deujsado" (26, A, 36); vo-lo, "nõ uollo 
consentira" (27, A, 69); vo-la, "que uolla possa dizer" (28, A, 21); (e) combinação/contracção com prep.: vosco, arc. (< vobiscum) = convosco, "uosco quero ẽtrar" $(29, \mathrm{~B}, 67)$; realizadas em port.: comigo, "vay comygo" (26, A, 63); commigo, arc.: "iunto cõ migo" (410, B, 21); contigo, "el he contigo" (412, A, 29); consigo, sing. e plur., "leuou com ssigo sua madre" (26, B, 66); "trazião consigo" (409, B, 24); connosco, "os que cõnosco quiserẽ ficar" (408, B, 42); convosco, "com uosco quero hir" ( $26, \mathrm{~B}, 29)$; del (<>de+el), arc., "açerca dell" (29, A, 50); dele (<>de+ele), "o que se fez dele" (418, A, 63); dela, "ouue della estes filhos" (22, A, 46); "filhauã dela [partitivo=alguma]" (413, A, 28); deles, "mjlhares delles" (25, B, 64); "segundo seu emtemder deles" (76, B, 74); nele (<>em+ele), "nele moreo" (416, A, 16); nela, "que nela leuarom" (417, A, 29); pelo ( $<$ per, ant.+lo), "pello homrar em seu casamento" (26, A, 30); polo, ant. (<>por+lo), "pollo fazer alleuantar" ( 27, A, 36).

B. Possessivos (pronomes (a) e adjectivos (b)): $1^{\mathrm{a}}$ p.: minha, "mjnha he a terra" (26, B, 21); $3^{\mathrm{a}}$ p.: os seus, "aos seus" (26, A, 69); sua, "que era ssua" (26, A, 41); (b) 1"a p.: meu, "por meu amor" (26, B, 30); a minha, arc., "a mjnha morada" (75, B, 62); os teus, "aos teus homẽẽs" (26, A, 58); (o) seu, "todo seu dotamẽto" (412, B, 20); "no seu moesteiro" (22, A, 53); os seus, "os seus Jujzes" (22, A, 23); (a) sua, "asy foy sua vida" (22, A, 52); "aa sua custa" (22, A, 54); suas, "suas companhas" (408, A, 4); nosso, "nosso señor Jesu" (22, A, 20); nossa, "nossa madre" (407, A, 32); vosso, "uosso filho" (26, B, 24); vossa, "uossa madre" (26, B, 39); deles, "segundo seu entender deles" (76, B, 74); [pleonasmo arc.]. Formas antigas dos adj. pos.: sa, "toda ssa terra" (27, B, 44); sas, "cõ ssas perteeças" (29, $\mathrm{B}, 17)$; as tas, "quebrantadas sejã as tas pernas" (30, A, 18).

C. Démonstrativos (pronomes e adjectivos: ambos, "danbos estes castellos" (25, B, 13); a, fem. sing., "aque chamarõ adelharcos" (24, A, 46); o, masc. sing., "o de mayor poder" (24, A, 68); "cõ $o$ que era gaanhado" (26, A, 34); os, masc. plur., "os que a leer ouuire [sic]" (407, A, 41); "os destorga" (26, A, 61); aaquel, arc. "aaquel que era" (410, B, 15); <> àquele; aaquela, arc., "aaquela palma" (413, A, 27); $<>$ àquela; aaqueles, arc., "aos franceses e aaqueles" (411, B, 41) <> àqueles; aquel, arc. <> àquele, "aquel tempo" (408, B, 8); "aquell que vem" (29, A, 15); aquele, "aquelle seu filho" (26, A, 45); aquela, "aquella sazom" (26, B, 9); aqueles, "aquelles cinquo Reis" (27, B, 60); "aqueles que erom" (76, B, 61); aquelas, "aquela(s) companhas" (418, B, 32); aquelo, arc. $<>$ aquilo, "aquelo que lhe ia por tantas uezes mandara fazer" (410, B, 29); aquilo (?), "que era aquello/aquillo" (27, A, 54) [O ms. traz aqllo, que tanto pode ser uma coisa como a outra; aquilo documenta-se já em 1214; cf. Huber, Altportug., §346; aqueste, ant.<> este, "Aqueste cardeall" (30, $\mathrm{A}, 65)$; aquesta, ant.<> esta, "aquesta mjnha molher" (27, B, 25); aquestas, ant. <> estas, "aquestas mããos" (27, B, 23); assa, arc. (=a essa < ăd ı̆psa-), "ismar, que assa sazom era Rey" (27, B, 50); asta, arc. (= a esta < ăd istă), "asta sazom quasou" (30, B, 48); esta, "Esta he arrenenbrança" (22, A, 17); "esta ujlla" (27, A, 17); este, "Este conde" (22, A, 38); "e este auja nome otha" (407, B, 29); estes, "estes filhos" (22, A, 46); "Estes erã muytos" (409, B, 52); estas, "estas eras" (22, A, 31); esto, ant. <> isto, "Esto nõ he apocrifo" (25, A, 56); 
56); neste, "neeste preito" (29, B, 58); esse, "en esse tempo" (407, B, 26); "esse que uos dizedes" (30, A, 50); essa, "ẽ essa egreia" (409, B, 9); esses, "todos esses" (412, A, 26); elo, arc. $<>$ isso, aquilo, "ffallarom ẽ ello" (28, B, 44); do (=daquilo), "auerdade doque" (22, A, $32)$; (=daquele), "deuaçom do que o deu" (76, B, 55); daquele, "memoria daquelle boo aqueçimento8 (27, B, 59); desse, "sobrinho desse Rey" (22, B, 23); desses, "os nomes desses marteres" (409, B, 35); deste, "deste lenho" (24, B, 57); destes, "fforom destes Regnos" (22, A, 17); desto, ant. (=isto), "ante desto" (29, A, 52); delo, ant. (=disso), "nõ curou delo" (410, B, 26).

D. Relativos: que, suj., "aaquel que era guardador" (410, B, 15); compl. dir., "que ell fundou" (22, A, 54); a que, compl. ind., "aque elle deu Villa de Conde" (22, B, 28); quem, suj., "quem vem" (417, A, 23); compl. ind., "a que mester fazia" (413, B, 24); o qual, " $O$ quall segũdo a dada dessa escriptura" (22, A, 27); a qual, "A qual rrenenbrança" (22, A, 21); os quaes, arc. (=os quais), "os quaaes forõ na filhada" (407, B, 28); as quaes, arc. (= as quais), "As quaees aqui som escriptas" (22, A, 33); pelo qual, "pelo quall era mujto amado" (75, B, 48); na qual, "na qual se faz cada dia memoria" (407, A, 33); dos quaes, arc. (=dos quais), "no sangue dos quaaes" (410, A, 47); onde, "onde Jaz sepultado" (22, A, 30); arc. (=donde), "terras honde eram e de que sangue uinham" (409, B, 36); donde, "donde trouxe mujtas Riquezas" (25, B, 60); aonde, ant. e pop. no sentido de onde, "daly a onde estauom" (417, A, 12); u, ant. (=onde), "per hu ell uem" ( $28, \mathrm{~A}, 35)$; quê, "nom avya rrazom por que" '( $(27, \mathrm{~B}, 31)$; cuija, arc. (=cuja), "Cujya alma deus aja" (78, B, 9); "Por cujya e semelhança [sic] do diuinal misteryo" (25, B, 1); cujo, "cuio logo uos teedes" (408, B, 62); cuja, "cuja alma deus aja" (76, B, 2); cujos, "cujos corpos forom despois tidos em gramde reliquia e reueremçia" (418, B, 14).

E. Interrogativos: (pron. e adjectivos): qual, "quall riqueza me trazedes [...]?" (28, A, 44); quaes, arc. (=quais), "maginou quaaes forom" (75, B, 63); quais, "notou muj bem quaajs erom os ofiçios" (75, B, 68); quanto, "quanto ben e mercee" (408, B, 11); quantos, "quãntos corpos" (413, B, 52); quantas, "quantas E quaees" (28, B, 25); que, "que gritos atam altos" (78, A, 18); quem, "quem pode declarar os muj altos sospiros deste santo homẽ" (77, B, 13).

F. Indefinidos: (pronomes e adj.): al, ant. (=outra coisa), "todo oall"' (26, A, 64); algo, "setrazedes algo" (30, B, 7); algum, "algum lugar" (416, B, 24); alguns, "alguns fariom"

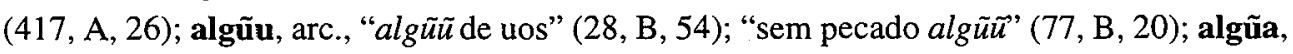

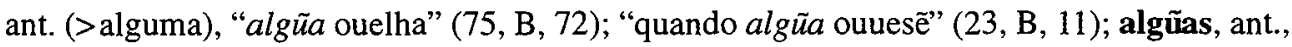
"algũas doaçõoes" (22, A, 23); algũus, arc., "outros alguũs" (413, A, 29); "algũüs emijgus" (76, A, 40); alguém, "alguem disse" (27, A, 16); atanta, arc. (=tanta), "virom atanta toruaçom" (77, A, 4); cada, "cadadia" (29, A, 74); cada ũu, arc. (=cada um), "cada hũu Rey" (22, A, 29); certo, "deulhe çerta (f.) terra" (26, A, 35); "çerto conde" (26, B, 19); nemigalha, arc. (=nada), "nom teuesse de veer hy nemjgalha" (77, A, 12); nem um, "nemhum tor-

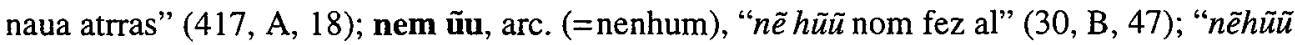
desaguisado" (27, B, 30); neũu, arc. (=nenhum), "ssem ssabendo nehüü" (27, A, 6); nem 
ũa, arc. (=nenhuma), "nẽhũa cousa" (26, A, 48); "nõ ouerõ colheita nẽ hüa" (408, A, 2); ninguém, "que o não semtio ninguem" (417, A, 1); nenguém, arc., "por nẽguem" (30, A, $44)$; outrem, "dado aoutrem" (27, A, 62); outro, "Eoutro em omoesteiro" (23, A, 6); "outro homẽ" (407, B, 27); outra, "sem outra detardança" (408, A, 33); outros, "Eoutros dous filhos" (23, A, 5); outras, "Eoutras escripturas" (22, A, 23); pouco, "a pouco tempo" (76, B, 29); pouca, "pouca diligencia" (24, B, 5); poucos, "huũs poucos de mouros" (408, A, 41); "poucos dias" (411, B, 27); qual, "tall enmenda quall ffor" (27, B, 26); quaes, arc. (=quais), "quaees elle quis" (28, B, 19); tal, "em tall guisa" (26, B, 75); taes, arc. (=tais), "fazendo taaes cartas" (22, A, 25); qualquer, "qualquer cousa" (410, A, 16); quaesquer, arc. (=quaisquer), "quaesquer gemtes" (419, B, 59); tanto, "tanto tempo" (25, B, 37); "alguũ tanto" (29, B, 23); tanta, "çõ tanta deuaçõ" (408, A, 35); tantos, "tantos que sse apoderarom" (29, A, 7); tantas, "tantas companhas" (408, A, 38); todo, "todo seu dotamẽto" (412, B, 20); "Todo uerdadeiro cristãão" (407, A, 23); toda, "toda a Jurdiçom" (24, A, 73); todos, "pera todos" (23, B, 8); todas, "todas as offertas" (409, B, 14); tôdalas, "todallas ffortellezas" (26, B, 65); tôdolas, arc., "todolas cousas" (419, B, 56); tôdolos, "todollos

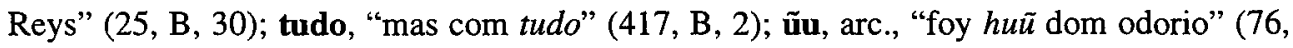
B, 34); ũus, arc., "ficarõ huũs poucos" (408, A, 41); uns, "hums com os outros" (416, $\mathrm{B}, 23)$;

Observação final. Aqui fica apenas uma amostra desta parte da morfologia da linguagem empregada em os nossos cronicões, ou seja a do período arcaico do português (nestes textos, séculos XIV e XV), começado no fim do século XII. O que há de mais notável é a grande semelhança entre a língua portuguesa desse tempo e a posterior, incluindo a actual, sobretudo na sua forma popular.

\section{MORFOLOGIJA ČLENA, ŠTEVNIKA IN ZAIMKA V PORTUGALSKIH KRONIKAH}

Avtor analizira morfologijo člena, števnika in zaimka, kot jo je mogoče ugotoviti pri kronistih, ki so soustvarjali stare portugalske kronike od XIII. do XV. stoletja, zbrane in objavljene v delu PORTUGALIAE MONUMENTA HISTORICA. Začenja s predstavitvijo določnega člena, zlasti v povezavah in stapljanju s predlogi. Za števnik ugotavlja, da so stare oblike iz kronik v evropski portugalščini kdaj pa kdaj šle iz rabe, pač pa se še najđejo v brazilski portugalščini. Pri osebnem zaimku so značilne razlike med naglašenimi in nenaglašenimi oblikami. Ene in druge najdemo celo ob glagolu. Pri svojilnih zaimkih je ugotovljiva tudi raba določnega člena, kadar je svojilni zaimek rabljen pridevniško, kar je tudi danes značilnost portugalskega jezika (ne pa španskega), vendar raba v kronikah ni čisto dosledna. 